\title{
Teste Palográfico: Desafios para Formação em Avaliação Psicológica
}

\author{
Eduarda Lehmann Bannach ${ }^{1}$ (D) Alessandra Sant'Anna Bianchi (D) \\ Universidade Federal do Paraná, Curitiba-PR, Brasil
}

\section{RESUMO}

O Teste Palográfico é um teste de personalidade muito utilizado no Brasil. Objetivou-se na presente pesquisa realizar uma análise sobre o modo que a correção deste teste vem sendo realizada por psicólogos que trabalham cotidianamente com avaliação psicológica. Para tanto, foram aplicados questionários em 295 psicólogos. Estes tinham entre 26 e 66 anos (média=40,34; DP=9,91), 94,9\% eram do sexo feminino. Os resultados indicam uma falta de padronização na correção do teste, o que gera a possibilidade de uma mesma pessoa receber diferentes pareceres, dependendo do critério utilizado. Além disso, três problemas foram encontrados: a rapidez na correção do teste; o não uso dos materiais necessários para corrigi-lo e o erro de significado atribuído aos indicadores. A partir dos erros de correção do teste e do pouco tempo utilizado nesse processo, discute-se a necessidade de que seja dada atenção à formação do psicólogo em avaliação psicológica.

Palavras-chave: teste psicológico, teste expressivo, graduação em Psicologia.

\section{ABSTRACT - Palographic test: Challenges for training in psychological evaluation}

The teste Palográfico (Palographic test) is a personality test used a lot in Brazil. The aim of this study was to perform an analysis on the way that correction of this test has been performed by psychologists that work daily with psychological evaluation. For this, questionnaires were applied to 295 licensed psychologists. These were aged between 26 and 66 years $(M=40.34, S D=9.91)$; with $94.9 \%$ females. The results highlight the lack of standardization in the correction process of the test, which generates the possibility of the same person receiving different results depending on the criteria used. In addition, three problems were found: the time spent during the correction process; lack of application of proper methods to evaluate the tests and the incorrect interpretation of test items. From the errors in the correction of the test, we discuss the need to consider the psychologist's training regarding psychological assessment.

Keywords: psychological test; expressive test; degree in psychology.

\section{RESUMEN - Test Palográfico: Retos para la formación en evaluación psicológica}

El Test Palográfico es un test de personalidad muy utilizado en Brasil. El objetivo de la presente investigación fue realizar un análisis sobre el modo en que la corrección de este test viene siendo efectuada por los psicólogos que trabajan diariamente con evaluación psicológica. Para ello, 295 psicólogos contestaron al cuestionario. Estos tenían entre 26 y 66 años (media=40,34, DS=9,91), siendo $94,9 \%$ mujeres. Los resultados indican una falta de estandarización en la corrección del test, lo que genera la posibilidad de que, dependiendo del criterio utilizado, una misma persona pueda recibir diferentes resultados. Además, se encontraron tres problemas: la rapidez en la corrección del test; los materiales necesarios para su corrección no fueron utilizados y el error de significado atribuido a los indicadores. A partir de esos resultados, se discute la necesidad fomentar la formación del psicólogo en evaluación psicológica. Palabras clave: test psicológico; test expresivo; graduación en Psicología.

Os testes psicológicos são ferramentas úteis por fornecerem informações importantes sobre as pessoas que estão sendo avaliadas, gerando mais subsídios para realizar a avaliação psicológica (Figueirêdo \& Moura, 2015). A avaliação psicológica, por sua vez, pode ser entendida como um processo que objetiva chegar a hipóteses ou diagnósticos sobre características de personalidade, aptidões para uma tarefa, funcionamento intelectual, entre outras possibilidades (Hutz, 2015). Esse processo deve ter suas conclusões baseadas na investigação científica (Conselho Federal de Psicologia, 2011). É preciso destacar que os testes psicológicos podem ser parte da avaliação psicológica, mas não é obrigatório que sejam aplicados (Hutz, 2015).

O Conselho Federal de Psicologia (CFP) (2006) apontou uma tendência nacional para a utilização do

Endereço para correspondência: Departamento de Psicologia, sala 214. Praça Santos Andrade, 50, Centro, 80020-300, Curitiba, PR. Tel.: (41) 3310-2649. E-mails: bianchi@ufpr.br; eduardabannach@gmail.com

Artigo derivado da Dissertação de mestrado de Eduarda Lehmann Bannach com orientação de Alessandra Sant'Anna Bianchi, defendida em 2019 no programa de pós-graduação em Psicologia da Universidade Federal do Paraná". 
Teste Palográfico como instrumento para avaliar personalidade no contexto de obtenção da Carteira Nacional de Habilitação $(\mathrm{CNH})$, por exemplo. Esse teste avalia autoestima, ânimo, humor, relacionamento interpessoal, agressão, organização, impulsividade, aspectos depressivos e outras características (Alves \& Esteves, 2009). O Teste Palográfico está com parecer favorável para uso, de acordo com o Sistema de Avaliação de Testes Psicológicos, desde 2004 (Conselho Federal de Psicologia, 2019a). Tal avaliação compreende aspectos quantitativos e qualitativos do teste. Reppold et al. (2018) afirmam que o elevado número de testes "projetivos/expressivos/gráficos" (sendo o Palográfico um teste expressivo de acordo com seu manual) aprovados pelo CFP significa um avanço na área, visto que demonstra que esses testes possuem rigor nos estudos de confiabilidade e validade.

Apesar de o Teste Palográfico ser amplamente utilizado, a produção científica sobre seus usos é escassa (Sampaio \& Nakano, 2011). Mognon e Rueda (2016) afirmam ser necessário que pesquisadores brasileiros desenvolvam estudos empíricos sobre avaliação de aspectos de personalidade.

Além disso, mesmo havendo dados no manual do Teste Palográfico (Alves \& Esteves, 2009) sobre quais indicadores representariam riscos para o trânsito (contendo as médias dos indicadores atingidas pelo grupo de motoristas com e sem acidentes), não há dados específicos sobre a partir de que valor determinado motoristas podem provocar situações de risco. É importante ressaltar, entretanto, que este não é o objetivo do teste. Na nova versão, publicada em 2019 (Alves \& Esteves, 2019), o mesmo estudo de 2004 é apresentado, ou seja, há apenas as médias para os dois grupos mencionados, mas não há um limite do que é seguro. Isso é um problema, visto que o Conselho Federal de Psicologia (2000) afirma que toda avaliação psicológica deve ser realizada a partir de um código operacional que permita que qualquer psicólogo chegue aos mesmos resultados em dado período. Uma das críticas aos testes psicológicos está justamente na falta de padronização para determinadas realidades e contextos (Calejon, 2007).

As Diretrizes Curriculares Nacionais para cursos de graduação de Psicologia afirmam que uma das competências do formado no curso é ter domínio básico de conhecimentos sobre avaliação de processos psicológicos de indivíduos (Brasil, 2011). Entretanto, segundo Ambiel, Zuanazzi, Sette, Costa e Cunha (2019), há muito o que se modificar para que o ensino de avaliação psicológica atinja uma qualidade mínima. As mudanças necessárias para haver uma prática qualificada dependem da qualidade da formação que os cursos de Psicologia oferecem (Borsa, 2016).

De acordo com o estudo de Bardagi, Teixeira, Segabinazi, Schelini e Nascimento (2015), as instituições de ensino não fornecem salas adequadas para a prática em avaliação psicológica e os materiais disponíveis muitas vezes estão em más condições ou desatualizados, fazendo com que a experiência dos alunos esteja distante da prática efetiva na área. Noronha, Carvalho, Miguel, Souza e Santos (2010) afirmam que a infraestrutura é um eixo de formação importante em avaliação psicológica, porém, é necessário prestar atenção no conteúdo das disciplinas.

Alguns desafios são elencados na formação do psicólogo em relação à avaliação psicológica, como a organização de um contexto de ensino que assegure uma reflexão crítica ao aluno, estímulos à compreensão sobre medidas em Psicologia (psicometria) e o lugar que estas ocupam no processo de avaliação e compreender os fundamentos teóricos dos instrumentos (Ambiel et al., 2019; Calejon, 2007). A análise sobre as disciplinas de avaliação psicológica da região Norte do Brasil, realizada por Freires, Silva Filho, Monteiro, Loureto e Gouveia (2017), mostra que a fundamentação em psicometria não é destaque nas ementas, sendo considerada um elemento secundário.

Outro problema é a elaboração do laudo psicológico, documento final do processo de avaliação psicológica, que "apresenta informações técnicas e científicas dos fenômenos psicológicos" (Conselho Federal de Psicologia, 2019b, p. 14). A maioria das denúncias feitas ao Conselho Regional de Psicologia do Paraná em relação aos psicólogos são sobre a produção de documentos, sendo a elaboração de laudos a maior parte destes (Conselho Regional de Psicologia do Paraná, 2018). Sobre isso, Preto e Fajardo (2015) afirmam que a qualidade da elaboração do laudo psicológico não deve ser um objetivo do psicólogo apenas por receio de ser processado, mas sim que o profissional busque por qualidade da comunicação com o destinatário do laudo. Assim, o laudo psicológico pode ser entendido como expressão da competência profissional (Lago, Yates, \& Bandeira, 2016; Preto \& Fajardo, 2015). O motivo desse problema pode estar relacionado à pesquisa de Ambiel et al. (2019), que mostra que uma das categorias menos presentes nas ementas de avaliação psicológica é a que se refere, entre outras coisas, à elaboração do laudo psicológico.

É importante destacar também alguns elementos positivos sobre a formação em avaliação psicológica. $\mathrm{O}$ estudo de Bardagi et al. (2015) mostrou que, entre os 93 docentes de avaliação psicológica que participaram da amostra, 68,5\% atuam profissionalmente na área e a maior parte destes é mestre ou doutor. De acordo com Wechsler, Hutz e Primi (2019), pesquisadores com uma melhor formação trazem benefícios para o ensino nos cursos de graduação. $\mathrm{O}$ docente ter formação e experiência profissional na área é importante para o aprendizado dos alunos (Noronha et al., 2010). Outro fato positivo pode ser verificado na estrutura curricular do curso de Psicologia da Universidade Federal de Ciências da Saúde de Porto Alegre, que conta com sete disciplinas obrigatórias sobre Avaliação Psicológica (Reppold \& Serafini, 2010).

Yamamoto, Silva e Zanelli (2010) mostram que a maioria dos psicólogos tem envolvimento com alguma forma de pós-graduação e sugerem que um dos motivos 
disso ocorrer pode ser o fato destes reconhecerem a debilidade em sua formação. Outra possível explicação para isso pode estar relacionada ao resultado de Costa, Araújo e Almeida (2016), que mostra que estudantes mais velhos (com idade igual ou superior a 23 anos) tendem a se preocupar mais com a qualidade de sua formação. Lago et al. (2016) entendem que a formação em Psicologia não é suficiente para o psicólogo que deseja trabalhar com avaliação psicológica. As autoras acreditam que a especialização em Avaliação Psicológica deveria ser exigência do CFP para os psicólogos que pretendem trabalhar na área. Borsa (2016) aponta que a formação continuada é uma importante ferramenta para diminuir o preconceito em relação à área, o qual está atrelado a problemas técnicos e éticos presentes na prática nessa área.

Os profissionais que atuam na área estão despreparados, segundo Borsa (2016), tanto em aspectos técnicos como em aspectos teóricos. Calejon (2007) afirma que é necessário e urgente prestar atenção no que ocorre na formação do psicólogo no contexto da avaliação psicológica, sendo inevitável voltar o olhar para as instituições que preparam os futuros psicólogos para o exercício profissional.

Este estudo, então, propõe-se a entender, por meio da aplicação de um questionário, como o Teste Palográfico está sendo corrigido por psicólogos no Paraná, ou seja, quais indicadores têm sido utilizados e de que forma a correção vem sendo realizada (tempo de correção e materiais utilizados), pois a correção deste teste é um processo detalhado e minucioso (Cardoso, Esteves, Silva, Arsuffi, \& Franzim Neto, 2014). Não foram encontrados estudos com objetivos similares a este utilizando as palavras "palográfico" e "correção" na base de dados SciELO e no portal da Capes (busca realizada em 4 de maio de 2019).

As hipóteses para o objetivo em questão são: 1. que os psicólogos não escolhem os mesmos indicadores entre eles para corrigir o Teste Palográfico, visto que não há orientações formais sobre isso e 2. há problemas na correção do teste em questão. Essa hipótese foi pensada porque, no ano de 2007, o CFP lançou uma resolução (CFP $\mathrm{n}^{\circ}$ 003/2007) que limitava o psicólogo a atender um máximo de dez candidatos ao exame psicológico para Carteira Nacional de Habilitação, por jornada de oito horas de trabalho (Conselho Federal de Psicologia, 2007). Entretanto, em 2013, o CFP revogou tal artigo por pedido dos próprios psicólogos (Conselho Federal de Psicologia, 2013), sendo que hoje alguns estados seguem tal regra e outros não. Nesse contexto, é importante lembrar que o CFP considera que o conhecimento da Psicologia seja consolidado com padrões éticos, técnicos e científicos (Conselho Federal de Psicologia, 2019b).

\section{Método}

\section{Participantes}

Participaram do estudo 295 psicólogos, entre 26 e 66 anos (média $=40,34 ; D P=9,91$ ), sendo $94,9 \%$ do sexo feminino. Tais profissionais trabalham com avaliação psicológica diariamente, incluindo a aplicação e correção de testes. Entre os participantes, há representantes das diversas microrregiões do estado do Paraná. Todos os psicólogos afirmam utilizar o Teste Palográfico, sendo que $77 \%$ utilizam sempre, $17 \%$ muitas vezes e $6 \%$ poucas vezes. Os participantes são formados há, em média, 14,46 anos $(D P=8,54)$, trabalham com avaliação psicológica há, em média, 9,79 anos $(D P=6,83)$ e todos têm no mínimo título de especialista.

\section{Instrumento}

Foi utilizado um questionário impresso, elaborado pelas pesquisadoras, contendo questões sociodemográficas (sexo, idade etc.) e referentes à correção do Teste Palográfico (Alves \& Esteves, 2009), como, por exemplo, sobre tempo e os materiais utilizados pelo profissional para corrigir o teste. Além disso, também foi perguntado quais indicadores o psicólogo leva em consideração e o que, em cada indicador, este considera. Tal questionário possui cinco questões abertas e seis fechadas e foi preenchido pelos próprios participantes.

\section{Procedimentos}

Os dados foram coletados no segundo semestre de 2018. Para coleta dos dados, foi realizado um curso sobre atualizações em avaliação psicológica aberto para psicólogos que trabalham nessa área. Os participantes do curso foram convidados a responder um questionário e informados que o tempo médio de resposta era de 20 minutos. Do total de profissionais convidados a participar da pesquisa (333), 38 se recusaram. Os psicólogos que aceitaram participar assinaram o Termo de Consentimento Livre e Esclarecido e foram orientados a não conversarem durante o período que estivessem respondendo ao questionário. A pesquisa foi aprovada anteriormente pelo Comitê de Ética em Pesquisa em seres humanos do setor de ciências da saúde/UFPR (número 2685132).

\section{Análise de Dados}

Os dados foram analisados utilizando análises de frequência e correlação.

\section{Resultados}

Os psicólogos foram perguntados sobre onde aprenderam a corrigir o Teste Palográfico, tendo cinco opções. Nessa questão, era possível marcar mais de uma alternativa. Os resultados são apresentados na Figura 1. Ao analisar apenas as respostas de quem assinalou "Curso de especialização" e "Graduação em Psicologia", é possível perceber que $45 \%$ (117) dos participantes marcaram as duas opções; 40,8\% (106) assinalaram apenas "Curso de especialização" e 14,2\% (37) assinalaram apenas "Graduação em Psicologia". 


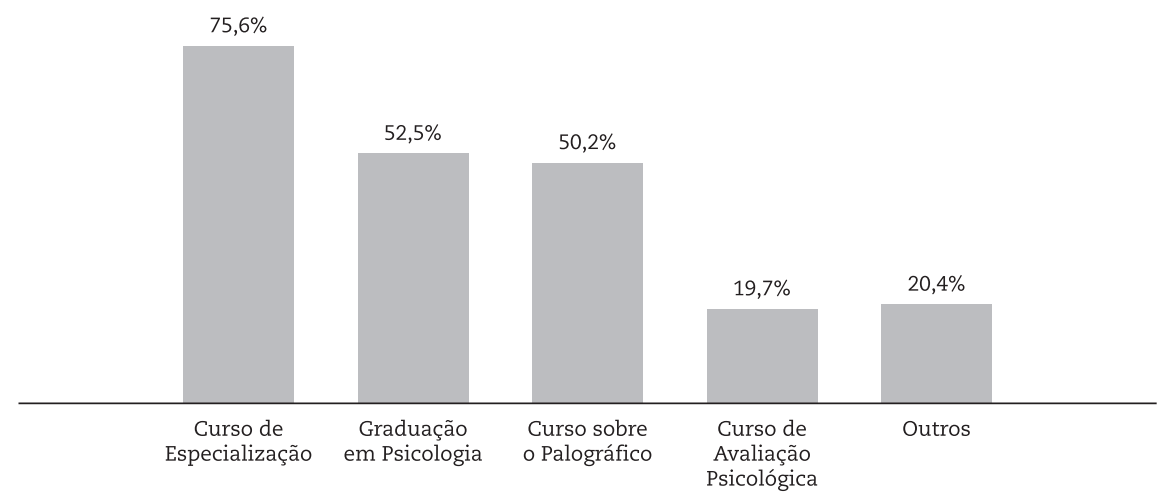

Figura 1. Onde os psicólogos aprenderam a corrigir o Teste Palográfico

Continuando sobre a correção do Teste Palográfico, foi perguntado aos profissionais quantos minutos, em média, levavam para corrigir cada teste. Como resultado, o tempo de correção variou entre dois e 120 minutos (média $=19,81$ minutos, $D P=14,1$ ). Outra inspeção sobre a distribuição dos tempos indicou que $71,2 \%$ dos psicólogos levam entre dois e 20 minutos para corrigir o teste e, além disso, $50 \%$ levavam até 15 minutos.

Em seguida, foi perguntado quais materiais e instrumentos os profissionais utilizavam para corrigir o teste. Essa era uma questão aberta, ou seja, não foram fornecidas opções. A régua $(74,4 \%)$, o manual $(51,1 \%)$ e a calculadora $(44,3 \%)$ foram os mais citados (Figura 2 ).

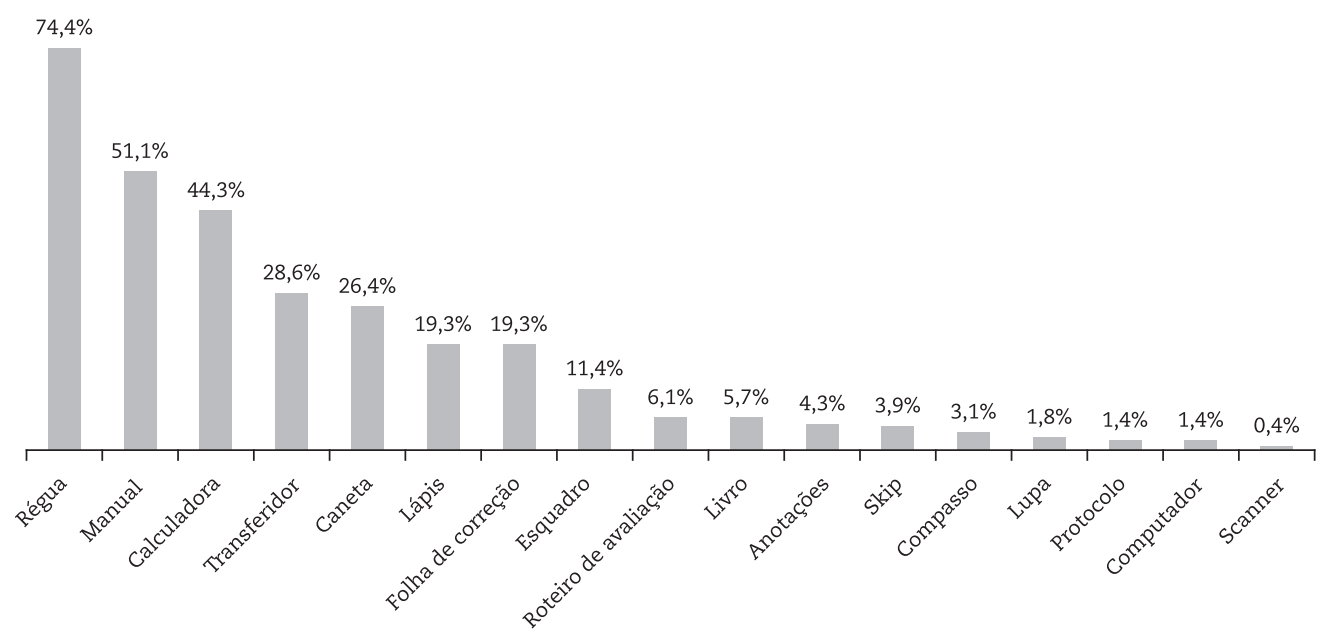

Figura 2. Materiais utilizados na correção do Teste Palográfico pelos psicólogos

Para corrigir determinados indicadores do Teste Palográfico, é imprescindível a utilização de alguns materiais. É o caso dos materiais que medem milímetros, como régua ou esquadro para correção dos indicadores: "Tamanho dos palos", "Distância entre palos", "Distância entre linhas", "Margem direita", "Margem esquerda" e "Margem superior"; e dos materiais que medem ângulo, como transferidor, para correção dos indicadores "Direção das linhas" e "Inclinação dos palos".

O uso da calculadora também é importante, visto que é necessário realizar contas de média em grande parte dos indicadores (normalmente com uma casa decimal), porém é possível que os profissionais realizem essa conta sem o instrumento. Caso o profissional opte por realizar a correção digitalizada, precisaria apenas do skip (Sistema de Correção Informatizada do Palográfico) e impressora contendo scanner. Entretanto, é possível notar que apenas 3,9\% dos psicólogos dizem utilizar o skip na correção.

Por isso, foi verificado se os profissionais que dizem corrigir determinados indicadores utilizam os materiais necessários para tanto (ver Tabela 1). É importante ressaltar que as pessoas que dizem utilizar o skip não foram contabilizadas na tabela em questão, visto que esse instrumento substitui a necessidade dos outros. Além disso, 
é relevante o fato de que as pessoas que mencionam utilizar o esquadro também mencionaram utilizar a régua.
Por isso, na Tabela 1 aparece somente a porcentagem de régua como instrumento que mede os milímetros.

Tabela 1

Porcentagem do Uso do Material entre os Psicólogos que Avaliam cada Indicador

\begin{tabular}{|c|c|c|c|}
\hline & Régua & Transferidor & Calculadora \\
\hline Tamanho dos palos & $77,6 \%$ & & $46,8 \%$ \\
\hline Distância entre os palos & $75,8 \%$ & & $47,2 \%$ \\
\hline Distância entre as linhas & $79,3 \%$ & & $46,2 \%$ \\
\hline Margem esquerda & $77,7 \%$ & & $48,2 \%$ \\
\hline Margem direita & $78,4 \%$ & & $43,9 \%$ \\
\hline Margem superior & $77,0 \%$ & & $44,9 \%$ \\
\hline Inclinação dos palos & & $32,7 \%$ & $46,2 \%$ \\
\hline Direção das linhas & & $29,9 \%$ & $48,9 \%$ \\
\hline
\end{tabular}

Quando perguntados sobre como foi feita a escolha dos indicadores do Teste Palográfico que eles utilizam para realizar a avaliação, $80,5 \%$ dizem ter consultado o manual do teste, como é possível ver na Figura 3. Foram dadas oito opções de resposta além da opção "Outros" e era possível marcar mais de uma opção.

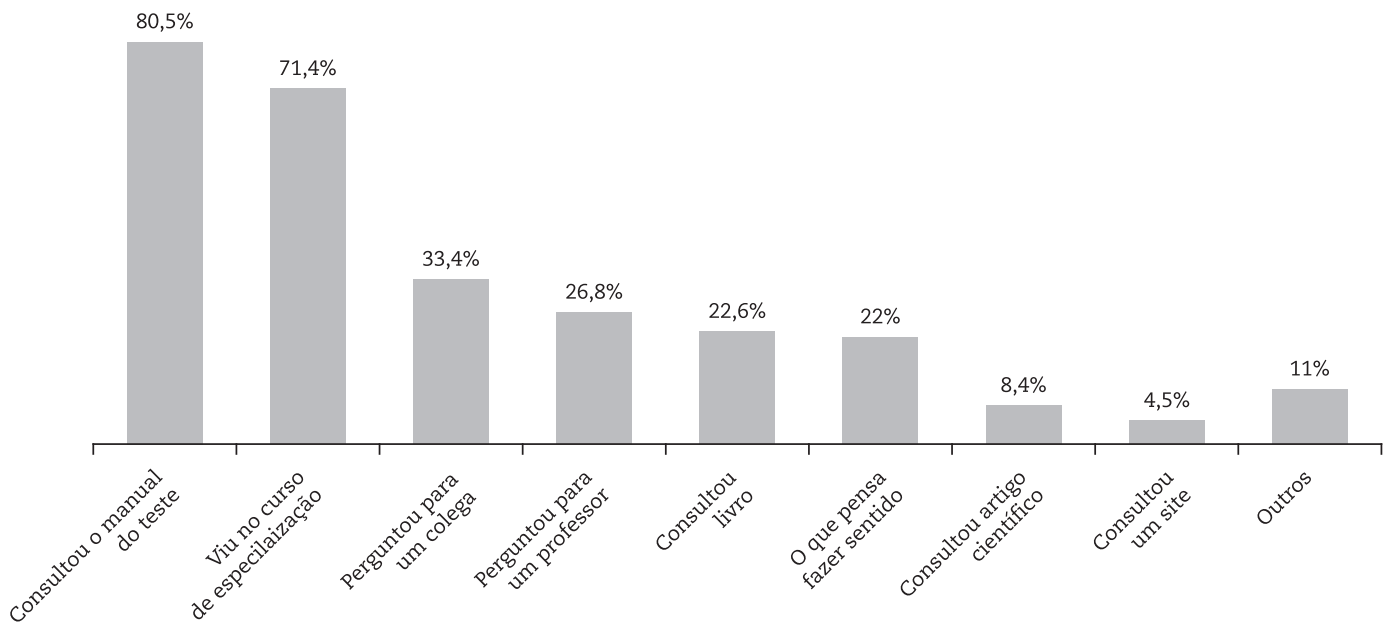

Figura 3. Como o profissional escolheu os indicadores do Palográfico para correção

No manual do Teste Palográfico, há 16 indicadores para correção. Cada um deles contém categorias, sendo que cada uma possui um significado. Os psicólogos deveriam marcar quais indicadores utilizam para corrigir o teste. Foram assinalados, em média, 10,33 indicadores. As frequências dos indicadores assinalados podem ser verificadas na Figura 4. Não houve correlação entre número de indicadores assinalados como corrigidos e tempo que os psicólogos levavam para corrigir o teste.

Depois de marcar o indicador que avaliavam deveriam responder, em cada indicador, o que analisavam. Ou seja, foram fornecidos todos os indicadores do teste, mas não foram fornecidas as categorias dos indicadores.
É preciso destacar que os psicólogos não estavam com o manual em mãos ao responder o questionário. Na Tabela 2, é possível verificar a frequência em que os psicólogos erravam o significado atribuído à categoria do indicador (coluna Erro) como, por exemplo, dizer que "Gancho" indica impulsividade sendo que, segundo o manual (Alves \& Esteves, 2009), indica agressividade. Essa frequência foi calculada levando em consideração somente o total de participantes que responderam avaliar aquele indicador e responderam algo sobre o que avaliavam no indicador (coluna Quantidade de respostas obtidas). Por exemplo: considerando as pessoas que responderam o que avaliavam no indicador Gancho, 8\% responderam de forma errada. 
Também é possível encontrar a frequência em que o psicólogo deixou de responder o que considerava em relação ao indicador em questão (coluna Resposta sem justificativa), embora o tenha assinalado. Essa frequência foi calculada considerando apenas as respostas que diziam considerar o indicador em questão, e não o total dos questionários.

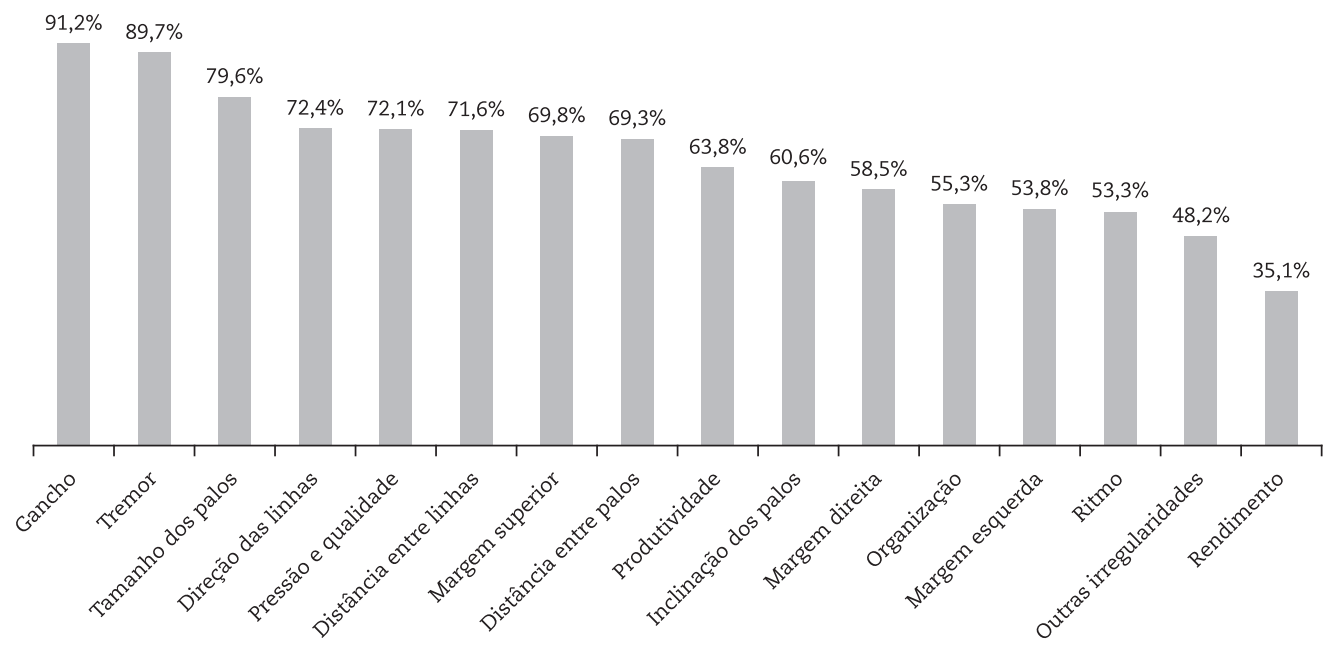

Figura 4. Frequência dos indicadores que os psicólogos levam em consideração

Tabela 2

Porcentagem de Respostas Erradas ou de Ausência de Resposta por Indicador

\begin{tabular}{lccc}
\hline \multicolumn{1}{c}{ Indicador } & $\begin{array}{c}\text { Respostas sem } \\
\text { justificativa }\end{array}$ & $\begin{array}{c}\text { Quantidade de } \\
\text { resposta obtidas }\end{array}$ & $\begin{array}{c}\text { Erro (calculado em relação } \\
\text { à coluna anterior) }\end{array}$ \\
\hline Gancho & $44,2 \%$ & 138 & $8 \%$ \\
Tremor & $46,9 \%$ & 130 & $4,6 \%$ \\
Tamanho dos palos & $42 \%$ & 127 & $3,1 \%$ \\
Direção das linhas & $49,7 \%$ & 100 & $2 \%$ \\
Pressão e qualidade do traçado & $51,5 \%$ & 95 & $14,7 \%$ \\
Distância entre linhas & $51,3 \%$ & 96 & $4,2 \%$ \\
Margem superior & $44,3 \%$ & 107 & $0 \%$ \\
Distância entre palos & $52,6 \%$ & 90 & $15,6 \%$ \\
Produtividade & $42,6 \%$ & 101 & $11,9 \%$ \\
Inclinação dos palos & $64,5 \%$ & 59 & $15,3 \%$ \\
Margem direita & $56,5 \%$ & 70 & $22,9 \%$ \\
Organização & $70,9 \%$ & 44 & $13,6 \%$ \\
Margem esquerda & $62,8 \%$ & 55 & $18,2 \%$ \\
Ritmo & $45,5 \%$ & 80 & $11,3 \%$ \\
Outras irregularidades do traçado & $72 \%$ & 37 & $24,3 \%$ \\
Rendimento & $88,7 \%$ & 11 & $63,3 \%$ \\
\hline
\end{tabular}

Nota. ${ }^{\text {a } O}$ indicador Rendimento é uma relação entre Produtividade e Ritmo, dessa forma se configura em um gráfico. Tal gráfico pode ser "horizontal", "equilibrado", rígido", "crescente", “decrescente”, em forma de "parabólica”, "côncava" ou "irregular”. Entretanto, a maioria das respostas dadas a essa questão era "muito baixo", possivelmente confundindo com o indicador "Produtividade"

Houve correlação significativa, utilizando a correlação de Pearson, sendo muito fraca e positiva, entre idade e tempo que o profissional leva para corrigir o Palográfico $(r=0,17, p \leq 0,01)$. Ou seja, quanto mais idade a pessoa tem, mais tempo leva para corrigir o teste. Além disso, houve correlação significativa e positiva, mas fraca, entre tempo de correção e tempo que a pessoa trabalha com avaliação psicológica $(r=0,2, p \leq 0,01)$. 


\section{Discussão}

O objetivo do presente estudo era realizar uma análise sobre o modo que a correção do Teste Palográfico vem acontecendo pelos psicólogos que o aplicam cotidianamente no Paraná. Uma das hipóteses iniciais é que não havia padrão em relação aos indicadores levados em consideração para correção. Essa hipótese foi corroborada, visto que nenhum indicador do Palográfico é escolhido por todos os profissionais e nenhum indicador é ignorado por todos os profissionais, ou seja, vários psicólogos não corrigem o teste por inteiro.

Apesar de uma das práticas mais desenvolvidas pelos professores de avaliação psicológica ser a correção de testes (Bardagi, Teixeira, Segabinazi, Schelini, \& Nascimento, 2015), a segunda hipótese, sobre haver problemas na correção do teste do Palográfico, também foi corroborada. Dois dados são preocupantes: o tempo que os psicólogos levam para corrigir o teste e os materiais que utilizam. O menor tempo relatado foi de dois minutos e a média foi de aproximadamente 20 minutos. Esse dado revela que muitos profissionais podem estar apenas olhando o teste rapidamente e não estão de fato corrigindo, visto que em dois minutos seria possível corrigir apenas o indicador Organização, que não necessita de operações matemáticas, porém, nenhum participante da pesquisa disse apenas corrigi-lo. Ao contrário, os psicólogos corrigem diversos indicadores (muitas vezes todos os indicadores possíveis). Assim, 20 minutos não seriam suficientes para correção, mesmo com experiência e agilidade, pois a maioria dos indicadores exige medidas ou porcentagens, resultando em contas matemáticas, o que demanda tempo.

Em relação às medidas, é possível apontar mais uma evidência de que alguns psicólogos não estavam realizando as medições necessárias, de acordo com o manual. Isso porque o número de profissionais que utiliza a régua para corrigir indicadores em que ela é necessária é menor que $100 \%$, ou seja, em média, $22 \%$ dos profissionais estão avaliando indicadores métricos sem régua, o que não é possível, segundo o manual. É importante lembrar que o Conselho Federal de Psicologia (2011) determina que sejam seguidas as orientações previstas no manual para analisar os dados. Além disso, uma das etapas do ensino de avaliação psicológica deve contemplar questões práticas como os materiais necessários (Noronha et al., 2010).

No caso dos indicadores que necessitam transferidor, os dados observados são ainda piores: menos de $33 \%$ das pessoas que dizem corrigir os indicadores em que medir ângulo é necessário citavam usar o material. Assim, em torno de $70 \%$ dos respondentes que corrigem tais indicadores não estão fazendo esse trabalho adequadamente. A falta de utilização dos materiais necessários encontrada mostra que o treinamento na aplicação e correção dos testes psicológicos é imprescindível para que o psicólogo tenha formação de qualidade, conforme exposto por Borsa (2016). É interessante que mesmo havendo suporte na literatura sobre altos índices de precisão do skip para medir variáveis quantitativas do Teste Palográfico (Cardoso et al., 2014), os participantes estão optando pouco por essa ferramenta $(3,9 \%)$, possivelmente por conta do seu custo financeiro.

Quanto ao lugar em que os psicólogos aprenderam a corrigir o teste Palográfico, o resultado mostra que a pós-graduação teve resultado mais expressivo que a graduação. Além disso, o curso sobre palográfico teve um resultado muito parecido com a porcentagem da graduação. Outro dado interessante é que mais de dois terços da amostra total $(n=106)$ diz ter aprendido a corrigir na pós-graduação sem assinalar ter aprendido na graduação. Dessa forma, parece que o profissional precisa compensar déficits em sua aprendizagem na graduação por meio de outros meios, o que vai ao encontro do que foi apontado por Yamamoto et al. (2010).

Ainda sobre esse tema, quando os psicólogos foram perguntados como escolheram os indicadores que corrigem no Palográfico, ter aprendido sobre o tema no curso de especialização aparece com a segunda maior frequência, revelando a importância da pós-graduação para os profissionais. Borsa (2016) enfatiza que a especialização se tornou um recurso essencial, visto que o que é aprendido na graduação não tem sido suficiente para oferecer os conhecimentos teóricos e práticos necessários para a atuação em avaliação psicológica. Tendo em vista a importância atribuída à especialização nas respostas da presente pesquisa, aponta-se esse espaço como interessante foco para uma possível intervenção futura. Ou seja, pode ser um interessante espaço para reforçar a ideia, para os psicólogos, sobre a importância de seguir as indicações do manual.

Chama atenção também o fato de apenas 8,4\% relatarem ter consultado artigos científicos para escolher os indicadores, sendo que $22 \%$ dizem escolher com base no que eles próprios pensam fazer sentido. Noronha et al. (2010) afirmam que é necessário que os psicólogos consumam leituras científicas, fazendo com que novos achados sejam aplicados na prática profissional.

Outra questão preocupante está na Tabela 2, ou seja, a grande quantidade de psicólogos que não responderam o que avaliam nos indicadores assinalados ou responderam errado. A princípio, os psicólogos não precisam saber tais dados sobre os significados dos indicadores com base em sua memória, pois há a possibilidade de consultar o manual. Entretanto, visto que a correção do Teste Palográfico é feita diariamente e em grande quantidade, chama atenção como tantas pessoas não souberam responder ou não acertaram a interpretação dos indicadores.

A questão que fica é: será que o Teste Palográfico está sendo corrigido de fato? E, caso esteja, será que a correção está sendo feita corretamente? Essa grande frequência de não resposta e erro remete à necessidade e 
urgência de prestar atenção no que está ocorrendo na formação do psicólogo em relação à avaliação psicológica (Bardagi et al., 2015; Borsa, 2016; Calejon, 2007). É necessário continuar analisando e implementando melhorias nas condições de ensino de avaliação psicológica na formação do psicólogo, de forma que este possa, no futuro, usar adequadamente os instrumentos existentes (Calejon, 2007).

A principal limitação deste estudo foi o fato de não acompanhar a correção do Teste Palográfico em si (medindo tempo, observando materiais utilizados e indicadores corrigidos), mas ter utilizado o autorrelato dos psicólogos sobre como fazem tal processo. Sempre é possível que haja diferenças entre o que é respondido e o que ocorre de fato.

A partir dos resultados da presente pesquisa, recomenda-se quatro importantes ênfases para os professores de disciplinas relacionadas ao ensino de avaliação psicológica, com a finalidade de melhorar a formação dos profissionais psicólogos. As recomendações são: 1. realizar um ensino de psicometria e fundamentos dos testes psicológicos aprofundado, antes de iniciar o ensino de aplicação e correção dos testes psicológicos; 2. haver a exaustiva explicação que o psicólogo deve seguir todas as regras do manual, tanto de aplicação, como de correção do teste, pois não seguir tais regras invalida os resultados encontrados; 3. explicar que os testes possuem materiais necessários para correção e que o profissional deve estar atento a quais são e deve utilizar todos corretamente e 4. ressaltar a necessidade da postura ética do psicólogo em relação à avaliação psicológica.

\section{Agradecimentos}

Não há menções.

\section{Financiamento}

Todas as fontes de financiamento para elaboração e produção do estudo (coleta, análise e interpretação dos dados, bem como, escrita dos resultados no presente no manuscrito) foram fornecidas pela Coordenação de Aperfeiçoamento de Pessoal de Nível Superior - Brasil (CAPES) - Código de Financiamento 001.

\section{Contribuições dos autores}

Declaramos que todos os autores participaram da elaboração do manuscrito. As autoras Eduarda Lehmann Bannach e Alessandra Sant'Anna Bianchi participaram da redação inicial do estudo - conceitualização, investigação, visualização, da análise dos dados e da redação final do trabalho - revisão e edição.

\section{Disponibilidade dos dados e materiais}

Todos os dados e sintaxes gerados e analisados durante esta pesquisa serão tratados com total sigilo devido às exigências do Comitê de Ética em Pesquisa em Seres Humanos. Porém, o conjunto de dados e sintaxes que apoiam as conclusões deste artigo estão disponíveis mediante razoável solicitação ao autor principal do estudo.

\section{Conflito de interesses}

Os autores declaram que não há conflitos de interesses.

\section{Referências}

Alves, I. C. B., \& Esteves, C. (2009). O teste palográfico na avaliação da personalidade. 2. ed. São Paulo: Vetor.

Alves, I. C. B., \& Esteves, C. (2019). O teste palográfico na avaliação da personalidade. 3. ed. São Paulo: Vetor.

Ambiel, R. A. M., Zuanazzi, A. C., Sette, C. P., Costa, A. R. L., \& Cunha, F. A. (2019). Análise de ementas de disciplinas de avaliação psicológica: Novos tempos, velhas questões. Avaliação Psicológica, 18(1), 21-30. doi: 10.15689/ap.2019.1801.15229.03

Bardagi, M. P., Teixeira, M. A. P., Segabinazi, J. D., Schelini, P. W., \& Nascimento, E. (2015). Ensino da avaliação psicológica no Brasil: Levantamento com docentes de diferentes regiões. Avaliação Psicológica, 14(2), 253-260. doi: 10.15689/ap.2015.1402.10

Borsa, J. C. (2016). Considerações sobre a formação e a prática em avaliação psicológica no Brasil. Temas em Psicologia, 24(1), 131-143. doi: 10.9788/TP2016.1-09

Brasil, Ministério da Educação, Conselho Nacional de Educação, Câmera de Educação Superior (2011). Diretrizes curriculares nacionais para os cursos de graduação em Psicologia. Resolução CNE/CES, no 5, de 15 de março. Brasília, DF. Recuperado de http://portal.mec. gov.br/index.php?option $=$ com docman\&view $=$ download\&alias $=7692$-rces005-11-pdf\&Itemid $=30192$

Calejon, L. M. C. (2007). Os desafios da avaliação psicológica na formação e exercício profissional do psicólogo. Em J. C. Alchieri (Eds.). Avaliação Psicológica: Perspectivas e Contextos (pp. 221-235). São Paulo: Vetor.

Cardoso, T., Esteves, C. Silva da, F. C., Arsuffi, E. S., \& Franzim Neto, L. (2014). Precisão do sistema de correção informatizada do teste Palográfico - Skip. Boletim de Psicologia, LXIV, 185-194. Recuperado de http://pepsic.bvsalud.org/pdf/bolpsi/v64n141/v64n141a07.pdf

Conselho Federal de Psicologia [CFP] (2000). Resolução nº. 012, de 20 de dezembro de 2000. Manual de avaliação psicológica de candidatos à Carteira Nacional de Habilitação e condutores de veículos automotores. Recuperado de https://site.cfp.org.br/wp-content/ uploads/2000/12/resolucao2000_12.pdf

Conselho Federal de Psicologia [CFPי] (2006). Relatório final da ação conjunta de fiscalização: Pesquisa exploratória sobre o processo de avaliação psicológica para obtenção da Carteira Nacional de Habilitação. Brasília. Recuperado de https://site.cfp.org.br/wp-content/ uploads/2007/05/relatorio_transito.pdf

Conselho Federal de Psicologia [CFP] (2007). Resolução nº. 003, de 12 de fevereiro de 2007. Institui a Consolidação das Resoluções do Conselho Federal de Psicologia. Recuperado de https://site.cfp.org.br/wp-content/uploads/2007/02/resolucao2007_3.pdf 
Conselho Federal de Psicologia [CFP] (2011). Resolução nº. 009, de 06 de maio de 2011. Altera a Resolução CFP nº 007/2009, publicada no DOU, Seção 1, do dia 31 de julho de 2009. Recuperado de https://site.cfp.org.br/wp-content/uploads/2011/05/resolucao2011_009.pdf

Conselho Federal de Psicologia [CFP] (2013, 7 de outubro). Esclarecimento sobre mudança na avaliação psicológica no contexto do trânsito. Recuperado de https://site.cfp.org.br/esclarecimento-sobre-mudanca-na-avaliacao-psicologica-no-contexto-do-transito/

Conselho Federal de Psicologia [CFP] (2019a). Testes favoráveis. Recuperado de http://satepsi.cfp.org.br/testesFavoraveis.cfm

Conselho Federal de Psicologian [CFP] (2019b). Resolução nº. 6, de 29 de março de 2019. Institui regras para a elaboração de documentos escritos produzidos pela(o) psicóloga(o) no exercício profissional e revoga a Resolução CFP n 15/1996, a Resolução CFP nº 07/2003 e a Resolução CFP no 04/2019. Recuperado de https://atosoficiais.com.br/cfp/resolucao-do-exercicio-profissional-n-6-2019-instituiregras-para-a-elaboracao-de-documentos-escritos-produzidos-pela-o-psicologa-o-no-exercicio-profissional-e-revoga-a-resolucaocfp-no-15-1996-a-resolucao-cfp-no-07-2003-e-a-resolucao-cfp-no-04-2019?q=006/2019

Conselho Regional de Psicologia do Paraná [CRP-PA] (2018). Caracterização dos processos disciplinares éticos indicados entre 2012 e 2017 no Conselho Regional de Psicologia do Paraná: relatório técnico. Curitiba: CRP-PR. Recuperado de https://crppr.org.br/wp-content/ uploads/2019/07/Relat\%C3\%B3rio-COE-25-06-19.pdf

Costa, A. R., Araújo, A. M., \& Almeida, L. S. (2016). Expectativas acadêmicas em estudantes de engenharia do primeiro ano: Uma análise de clusters. Psicologia, Educação e Cultura, 20(1), 65-78. Recuperado de http://pec. ispgaya.pt/edicoes/2016/PEC2016N1/index.html

Figueirêdo, M. L. R., \& Moura, G. C. (2015). A monitoria em técnicas de exames psicológicos II: contribuições, aprendizados e desafios. Cadernos de Graduação, 2(3), 173-185. Recuperado de https://periodicos.set.edu.br/index.php/fitshumanas/article/view/2028

Freires, L. A., Silva Filho, J. H., Monteiro, R. P., Loureto, G. D. L., \& Gouveia, V. V. (2017). Ensino da avaliação psicológica no Norte brasileiro: Analisando as ementas das disciplinas. Avaliação Psicológica, 16(2), 200-214. doi 10.10689/AP.2017.1602.11

Hutz, C. S. (2015). O que é avaliação psicológica - método, técnicas e testes. Em C. S. Hutz, D. R. Bandeira \& C. M. Trentini (Eds.). Psicometria (pp.11-21). Porto Alegre: Artmed.

Lago, V. M., Yates, D. B., \& Bandeira, D. R. (2016). Elaboração de documentos psicológicos: Considerações críticas à resolução CFP nº07/2003. Temas em Psicologia, 24(2), 271-786. doi: 10.9788/TP2016.2-20

Mognon, J. F., \& Rueda, F. J. M. (2016). Avaliação da personalidade no contexto do trânsito: Revisão de literatura. Avaliação Psicológica, 15(4), 33-43. doi: 10.15689/ap.2016.15ee.04

Noronha, A. P. P., Carvalho de, L. F., Miguel, F. K., Souza de, M. S., \& Santos, M. A. (2010). Sobre o ensino em avaliação psicológica. Avaliação Psicológica, 9(1), 139-146 139. Recuperado de http://pepsic.bvsalud.org/pdf/avp/v9n1/v9n1a15.pdf

Preto, C. R. S., \& Fajardo, R. S. (2015). Laudo psicológico no Brasil: Revisão da literatura com foco em estruturação e conteúdo. Archives of Health Investigation, 4(2), 40-52. Recuperado de http:/www.archhealthinvestigation.com.br/ArcHI/article/view/888

Reppold, C. T., \& Serafini, A. J. (2010). Novas tendências no ensino da avaliação psicológica. Avaliação Psicológica, 9(2), $323-329$ 323. Recuperado de http://pepsic.bvsalud.org/pdf/avp/v9n2/v9n2a16.pdf

Reppold, C. T. et al. (2018). Análise de manuais de testes psicológicos aprovados pelo Satepsi para avaliação de adultos. Revista Psicologia: Teoria e Prática, 20(3), 100-120. doi: 10.5935/1980-6906/psicologia.v20n3p100-120

Sampaio, M. H. L., \& Nakano, T. C. (2011). Avaliação psicológica no contexto do trânsito: Revisão de pesquisas brasileiras. Psicologia: Teoria e Prática, 13(1), 15-33. Recuperado de http://pepsic.bvsalud.org/pdf/ptp/v13n1/v13n1a02.pdf

Wechsler, S. M., Hutz, C. S., \& Primi, R. (2019). O desenvolvimento da avaliação psicológica no Brasil: Avanços históricos e desafios. Avaliação Psicológica, 18(2), 121-128. doi: 10.15689/ap.2019.1802.15466.02

Yamamoto, O. H., Silva, J. A. J. S. N., \& Zanelli, J. C. (2010). A formação básica, pós-graduada e complementar do psicólogo. Em A. V. B. Bastos \& S. M. G. Gondim (Eds.). O trabalho do psicólogo no Brasil (pp.45-65). Porto Alegre: Artmed.

\section{Sobre as autoras}

Eduarda Lehmann Bannach é psicóloga e mestre em Psicologia pela Universidade Federal do Paraná. Atualmente, é professora no curso de graduação em Psicologia da Universidade Estadual do Centro-Oeste.

Alessandra Sant'Anna Bianchi é psicóloga pela Universidade Federal do Rio Grande do Sul e doutora em Psicologia pela Universidad de Barcelona, Espanha. Atualmente, é professora nos cursos de graduação e pós-graduação em Psicologia da Universidade Federal do Paraná e coordenadora do grupo de pesquisa Trânsito e Transporte Sustentável (CNPq).

\section{Como citar este artigo}

Bannach, E. L., \& Bianchi, A., S. (2020). Teste Palográfico: Desafios para Formação em Avaliação Psicológica. Avaliação Psicológica, 19(4), 400-408. http://dx.doi.org/10.15689/ap.2020.1904.18487.06 